\title{
Editorial
}

\section{Coupled Flow and Heat or Mass Transfer}

\author{
Dimitrios V. Papavassiliou * , Sepideh Razavi ${ }^{(}$and Quoc Nguyen \\ School of Chemical, Biological, and Materials Engineering, The University of Oklahoma, Norman, OK 73019, \\ USA; srazavi@ou.edu (S.R.); qnguyen1010@gmail.com (Q.N.) \\ * Correspondence: dvpapava@ou.edu; Tel.: +1-405-325-5811
}

Received: 21 April 2020; Accepted: 24 April 2020; Published: 1 May 2020

Keywords: multi-phase flow; complex fluids; reactive flows; bioflows; computational fluid mechanics

About three years ago we invited contributions for a Fluids Special Issue on "Flow and Heat or Mass Transfer for the Chemical Process Industry." [1] There was a strong response from the community, leading to the publication of 13 papers in the areas of microfluidics, bio-applications, large-scale industrial flow applications, two-phase flows, and turbulent flows. This success indicated the interest of fluid dynamics researchers in investigating flow phenomena coupled with heat and/or mass transport for manufacturing and equipment design in emerging technologies. As a follow-up to that issue, we solicited contributions in the more general area of coupled flow and transport processes with industrial and environmental applications. The motivation for a second special issue stemmed from the fact that flows for energy production, manufacturing, materials processing, water treatment, food industry, and in the human body are most often coupled with heat or mass transfer. While progress in each individual area is significant, there is a need for systematic, rigorous investigation of this coupling of momentum, heat, and mass transport that is not only important for improving our overall theoretical understanding of transport phenomena, but is critical for the design of new materials, effective drug delivery, remediation of fracking sites, and engineering of novel, efficient processes. As we noted in the editorial of the first issue, "Generalizations and empiricisms have served practical needs in prior decades, but such empiricisms can now be revised or altogether replaced by understanding the interplay between flow and transfer." These generalizations and empiricisms have been epitomized in the use of overall transfer coefficients and effective transport properties; however, modern experimental and computational tools can yield in deeply understanding the coupling of flow and transport. The patterns of flow play a critical role in enhancing the transport of heat and mass. Typical examples are the coherent flow structures in turbulent boundary layers, and the flow patterns that are a function of the configuration of a porous medium and responsible for transport in a packed bed reactor or a separator unit.

The goal of this volume is to collect and present recent developments in theory and state-of-the-art experiments and computations on the interactions between flow and transport in complex flows and fluids, and in multiple length and time scales, which can be of significance for industrial and environmental transport processes. It includes papers that cover microfluidics, biological fluid mechanics, multiphase and reactive flows, and technical applications.

In microfluidics, Theodorakis et al. [2] reviewed recent molecular-level simulation techniques for the probing of the rapid and complete spreading of surfactant-laden droplets on hydrophobic surfaces-a phenomenon known for many years as superspreading, but only recently tackled with molecular simulations. The thorough character of this review makes it valuable for investigators in coating technologies, and also to investigators interested in advancing the field of fluid-substrate interactions with computations, since it identified the bottlenecks that require further research. Yamada et al. [3] is another numerical study on the use of coarse-grained techniques (Dissipative Particle Dynamics, DPD) in conjunction with a third-order Runge-Kutta scheme in order to reduce 
errors in thermal simulations. Molecular dynamics (MD) was used as the research tool to investigate the role of microemulsion on the recovery of hydrocarbons under confinement (nanoscale pore size) in the contribution of Bui et al. [4]. The behavior of oil, water, and surfactant molecules in tight pores that are common in shale rocks was probed to determine the effect of viscous forces on oil mobilization. While Theodorakis et al. [2], Yamada et al. [3], and Bui et al. [4] utilized molecular level numerical techniques, such as MD and DPD, Vlachomitrou \& Pelekasis [5] and Dobri \& Papathanasiou [6] employed numerical techniques that are appropriate for larger scales. Vlachomitrou \& Pelekasis [5] investigated the unsteady flow of a liquid metal inside a single pore in the presence of magnetic forces with a numerical approach. They identified the critical magnetic Bond number at which the liquid metal flow arrives at a steady state, and the critical number beyond which a Rayleigh-Taylor instability develops. They further investigated the dynamic response of different fluids, and the stabilizing effects of electrical conductivity and surface tension. A multi-scale numerical approach, based on hybrid numerical methods, for the multi-scale investigation for reactive flows in the space between fibers is presented in Dobri \& Papathanasiou [6].

In bio-applications, Ray and Heys [7] reviewed recent developments in our understanding of the mechanism that the brain uses to remove waste chemicals, toxic proteins, and biological debris. While the brain lacks lymph vessels to assist in removal of interstitial waste, it is efficient in doing so. Dysfunction of the brain's waste-removal system is correlated to disease, like Alzheimer's disease, traumatic brain injury, diabetes, and stroke. The review was focused on transport processes, especially the role that diffusion and advective flow play in the brain waste-removal process. Hard tissue in bone was the theme of Felder et al. [8]. Tissue engineered bone grafts are used to alleviate immunological response in allogenic grafts. Bone-tissue-engineered constructs are a logical approach to combat the issues commonly encountered with these bone grafting techniques. Felder et al. [8] examined oxygen uptake rates of mesenchymal stem cells seeded on poly(L-lactic acid) scaffolds cultured in a flow perfusion bioreactor to expand on current predictive metrics for the determination of bone construct characteristics, such as cellularity and cell type.

Multiphase flow phenomena were the theme of the next group of papers. Barkai et al. [9] examined the gas holdup, the superficial gas velocity, and the bubble size for the gas-liquid flow in large scale experiments in a vacuum airlift column. Mohagheghian et al. [10] investigated two-phase flow phenomena in another type of bench-scale column; a sparged bubble column. The goal was to explore the enhancement of mixing in the column by tracking the distribution of a passive scalar within a sparged swarm of bubbles. Experiments were used to compare mixing in static and vertically vibrating columns, indicating that in lower vibration amplitudes, vibration decelerated the mixing due to bubble retardation. Dimensional analysis showed that the non-dimensional mixing time is correlated to the transient buoyancy number. In the same area of bubble columns, Rek [11] presented a numerical simulation of the rising bubble in a stagnant liquid column. Open source Computational Fluid Dynamics software available through OpenFOAM was used to demonstrate that dynamic numerical meshes are more appropriate for predicting bubble trajectories. Moving to particle-fluid flows, Zaw et al. [12] employed Eulerian-Lagrangian simulation techniques to study particle deposition on the wall of a ribbed channel. The interplay between shear forces acting on the particles and the geometric configuration of the ribs, expressed as pitch to height ratio for the ribs, was investigated to find that the surface topography is important for mitigating particulate fouling of surfaces. Bratsun \& Vyatkin [13] analyzed theoretically selected problems in chemo- and thermo-vibrational convection. They derived exact non-steady, particular solutions to the Navier-Stokes equations. The novelty was in examining cases where the presence of a scalar field (temperature or concentration) made the hydrodynamic system spatially inhomogeneous.

In technological applications with industrial interest, Wei et al. [14] carried out a comparison between experiments and simulations for microjet cooling systems. The flow effects coupled with heat transfer are important for heat removal from electronic devices with liquid microjet impingement. Different numerical models for the turbulent flow environment were examined, and the Shear Stress 
Transport (SST) model and k- $\omega$ SST models showed the best ability to predict the thermal and flow field. In the same area of thermal fluids, Zhen et al. [15] presented the process of designing eco-friendly cellulose fibers from recycled paper waste. The waste is used to produce aerogels for thermal insulation, delivering a practical, engineering solution to enhance the heat insulation performance of water bottles, while not adding significant weight to the bottle. The effects of the static and dynamic conditions on heat flux were experimentally quantified. The paper that closes the volume deals with the evolution of organic fouling layers during operation of spiral wound desalination-membrane modules. Kostoglou \& Karabelas [16] presented a new approach to model fouling layer thickness evolution considering two types of non-uniform fouling; topological (induced by trans-membrane pressure variation) and non-topological (induced by membrane permeability variation). They proposed a methodology for the dynamic simulation of fouling at the membrane sheet scale. The paper is instrumental in developing general models and reliable simulators for membrane-desalination processes.

Finally, we thank the contributors to this volume for sharing their research, we thank the reviewers for generously donating their time to select and improve the manuscripts, and we thank the MDPI publishing and editorial staff for working with us to make this volume a success.

Conflicts of Interest: The authors declare no conflict of interest.

\section{References}

1. Papavassiliou, D.V.; Nguyen, Q. (Eds.) Flow and Heat or Mass Transfer for the Chemical Process Industry; MDPI: Basel, Switzerland, 2018; ISBN1 978-3-03897-238-9 (Pbk). ISBN2 978-3-03897-239-6 (PDF). [CrossRef]

2. Theodorakis, P.E.; Smith, E.R.; Craster, R.V.; Müller, E.A.; Matar, O.K. Molecular Dynamics Simulation of the Superspreading of Surfactant-Laden Droplets. A Review. Fluids 2019, 4, 176. [CrossRef]

3. Yamada, T.; Itoh, S.; Morinishi, Y.; Tamano, S. Temperature Error Reduction of DPD Fluid by Using Partitioned Runge-Kutta Time Integration Schemer. Fluids 2019, 4, 156. [CrossRef]

4. Bui, K.; Akkutlu, I.Y.; Silas, J.A. Mass and Momentum Transfer Considerations for Oil Displacement in Source Rocks Using Microemulsion Solutions. Fluids 2020, 5, 44. [CrossRef]

5. Vlachomitrou, M.; Pelekasis, N. Numerical Study of a Liquid Metal Oscillating inside a Pore in the Presence of Lorentz and Capillary Forces. Fluids 2020, 5, 44. [CrossRef]

6. Dobri, A.; Papathanasiou, T.D. Multi-Scale Modeling of the Dynamics of a Fibrous Reactor: Use of an Analytical Solution at the Micro-Scale to Avoid the Spatial Discretization of the Intra-Fiber Space. Fluids 2020, 5, 3. [CrossRef]

7. Ray, L.A.; Heys, J.J. Fluid Flow and Mass Transport in Brain Tissue. Fluids 2019, 4, 196. [CrossRef]

8. Felder, M.L.; Simmons, A.D.; Shambaugh, R.L.; Sikavitsas, V.I. Effects of Flow Rate on Mesenchymal Stem Cell Oxygen Consumption Rates in 3D Bone-Tissue-Engineered Constructs Cultured in Perfusion Bioreactor Systems. Fluids 2020, 5, 30. [CrossRef]

9. Barkai, A.H.; El Hajem, M.; Lacassagne, T.; Champagne, J.-Y. Experimental Study of a Gas-Liquid Flow in Vacuum Air-Lift Column Using an Optical Bi-Probe. Fluids 2019, 4, 145. [CrossRef]

10. Mohagheghian, S.; Ghajar, A.J.; Elbing, B. Effect of Vertical Vibration on the Mixing Time of a Passive Scalar in a Sparged Bubble Column Reactor. Fluids 2020, 5, 6. [CrossRef]

11. Rek, Z. Using a Dynamic and Constant Mesh in Numerical Simulation of the Free-Rising Bubble. Fluids 2019, 4, 38. [CrossRef]

12. Zaw, M.M.; Zhu, L.; Ma, R. Effect of Surface Topography on Particle Deposition from Liquid Suspensions in Channel Flow. Fluids 2020, 5, 8. [CrossRef]

13. Bratsun, D.; Vyatkin, V. Closed-Form Non-Stationary Solutions for Thermo and Chemovibrational Viscous Flows. Fluids 2019, 4, 175. [CrossRef]

14. Wei, T.; Oprins, H.; Cherman, V.; Beyne, E.; Baelmans, M. Conjugate Heat Transfer and Fluid Flow Modeling for Liquid Microjet Impingement Cooling with Alternating Feeding and Draining Channels. Fluids 2019, 4, 145. [CrossRef] 
15. Zhen, L.W.; Thai, Q.B.; Nguyen, T.X.; Le, D.K.; Lee, J.K.W.; Xiang, Y.Q.; Duong, H.M. Recycled Cellulose Aerogels from Paper Waste for a Heat Insulation Design of Canteen Bottles. Fluids 2019, 4, 174. [CrossRef]

16. Kostoglou, M.; Karabelas, A. Toward Incorporation of Membrane Properties Non-Uniformity in Spiral Wound Module Performance Simulators-Effect of Non-Uniform Permeability on Fouling Layer Evolution. Fluids 2019, 4, 127. [CrossRef]

(C) 2020 by the authors. Licensee MDPI, Basel, Switzerland. This article is an open access article distributed under the terms and conditions of the Creative Commons Attribution (CC BY) license (http://creativecommons.org/licenses/by/4.0/). 